\title{
Arcing Behaviour of A Potential High-Temperature Superconductor (HTS) Circuit Breaker Arc Model
}

\author{
A. Ullah, T.T. Lie, K. Gunawardane \\ Department of Electrical and Electronic Engineering \\ Auckland University of Technology (AUT) \\ Auckland, New Zealand.
}

\author{
N.K.C. Nair \\ Department of Electrical and Computer Engineering \\ The University of Auckland \\ Auckland, New Zealand
}

\begin{abstract}
Circuit breaker is vital and sophisticated part of protection accessories in power system. An arc model of a circuit breaker can determine the dynamic and static feature for arcing directed by a power circuit. The idea of HTS Circuit Breaker (HTSCB) evolves with the concept of HTS contacts in liquid nitrogen arc medium. Browne's HTS arc model for HTSCB and its characteristics developed and investigated earlier in order to address the arcing limitations of existing circuit breakers for HTS power applications. The model is mainly developed from the classical arcing equations. In this paper, the same arc model is investigated for the functionality of arc characteristics simulation response. The arc model has closed relationship with time constant, reference voltage and other parameters. The parameter sweep technique for minimizing the arcing time of HTS arc model parameters shows the trend of arcing voltage and current from the change of arc model's parameters. By using the final parameter values a test bed environment is developed in MATLAB ${ }^{\circ}$ Simulink to perform the interruption scenario of HTSCB. The results deal with different arcing terms like arc current peak, arcing time, fault duration, transient results of HTS arc model and the wave form transition tendency. Finally, the results are compared with the experimental analysis. It is discovered that there is significant level of agreement between experimental and simulated results.
\end{abstract}

Index Terms - Circuit Breaker, DC-AC inverter, arc model, power conditioning system, Arc Model.

\section{INTRODUCTION}

The physics of HTS has been extensively researched and used as commercial prototypes during the 21 st century. Recently, there has been interest emerging amongst researchers on the use of HTS for power system equipment developments. These remarkable developments of HTS application for power industry has introduced numerous prospective aspects with new pathway of research. This covers research areas such as $\mathrm{Bi}_{2} \mathrm{Sr}_{2} \mathrm{Ca}_{2} \mathrm{Cu}_{3} \mathrm{O}_{10+x}$ series HTS product development, HTS magnets and systems, methods, alongside large-scale manufacturing strategies and techniques. The companies like General Cable Superconductors Ltd., HTS-110 Ltd. etc. are supporting the comprehensive research for commercial rollout. Other institutes like Industrial Research Ltd. (IRL) and Robinson Research Institute [1] also have pivotal rule for the development of these technologies. The study of HTS cables, HTS transformers [2, 3], HTS wind turbine generators and HTS Fault Current Limiter [4], HTS external rotor machine [5] have been developed and trialed.
The investigation of HTSCB is therefore highly relevant and impactful in the context of additional development of HTS implementation pathway. The electrical behavior of HTSCB can be predicted by an arc model. An HTS breaker arc model with complex physical operations of HTSCB is developed earlier [6]. The prescribed arc model demonstrates described the arcing scenario when the contacts of HTCB makes the separation. Further investigation of the model with an HTS transformer shows significant advantage of very low Rate of Rise of Re-striking voltage as well [7]. The simulation data displayed an acceptable agreement for future implementation.

The major difficulties of arc simulation is to determine the time varying parameters. In this paper, a parameter sweep technique is carried out initially in order to investigate arc voltage, current and resistance for the variation of arc model parameters. The iterative simulation shows the transient response results of HTS arc model. The results are finally compared with the experimental analysis.

\section{DEVELOPMENT OF HTS BLACK BOX ARC MODEL}

Arcing is the major concern of the interruption procedure. The arc movement is identified as thermal plasma that is manifested by thermodynamic and electromagnetic principles. The research of arcing characteristics and numerous arc models are performed for years in order to comprehend arc behaviour. A physical arc model is often incredibly complex to operate. An arc model can be utilised to investigate overall arc behaviour in details throughout the interruption [6].

The basic objective of the proposed black box arc model is to determine the relationship between the electrical circuit and the arc during interruption procedure. The black box model represents non-linear arc conductance variation with respect to time. The models are described by first or second order differential equations that are developed with assumptions in order to streamline arc physics. The black box arc model comes with the hypothesis of local and global thermal equilibrium. It also includes the theory of energy conservation for arcing scenario. This is important to determine the best optimized values for the parameters of black box arc model in accordance to the experiment results. The primary results of arc model usually predicts the arc current and voltage behaviour. A Browne's model for HTS application and its calculation of power system transients is performed using MATLAB ${ }^{\circledR}$ 
Simulink program. In this proposed model the HTS breaker mechanism is maintained with a time constant of first order [6].

\section{A. Fault Analysis}

The HTS arc model describes electrical arcing, the current interruption and the steady state voltage and by steadily change the arc conductivity. The setup is modelled as a frequency dependent model. When $11 \mathrm{kV}$ supply is applied, it results in a high current that exceeds the circuit breaker current rating and hence the breaker trips. The reset is then pressed, and the voltage is ramped up. The breaker trips beyond the circuit breaker current rating. This is the simple mechanism for the HTS arc model. A device block is used to switch the differential into physical measurement signals pressure, $\mathrm{P}$ and temperature, $T$. In order to use the breaker especially for other HTS application, the MATLAB model assumes an ideal pressure and temperature sensor. The pressure and temperature are assessed across two ports of the HTS breaker arc model.
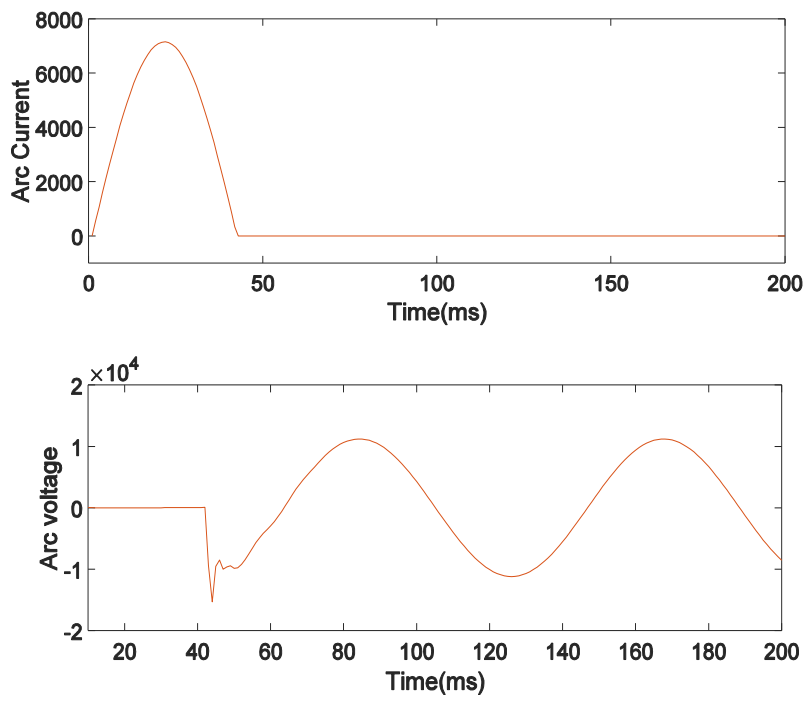

Figure 1: Arc voltage and current for HTS Arc model [6]

Figure 1 shows the short circuit fault result for the HTS arc model. A very high current is tripped within 50 millisecond with the Browne's HTS arc model. The arc model is based upon two major arc equations. Browne simplified the equation of Cassie and the Mayr. The new two equations are followed as:

a) Cassie's theory prior to current zero as:

$$
\frac{d}{d x}\left(\frac{1}{R^{2}}\right)+\frac{2}{\theta}\left(\frac{1}{R^{2}}\right)=\frac{2}{\theta}\left(\frac{1}{E_{0}}\right)^{2}
$$

b) Mayr's theory around current zero as:

$$
\frac{d R}{d t}-\frac{R}{\theta}=-\frac{e^{2}}{\theta N_{0}}
$$

The differential equations prior to and around current zero are modeled in MATLAB ${ }^{\circledR}$ Simulink. This study helps to develop a precise mathematical model to determine and sum up the specific behavior of HTS switch arc. Moreover, it helps in focusing the chance of applying this model into the real-time
Supervisory Control And Sata Acquisition (SCADA) system. These differential equations are solved using ODE solver in MATLAB for 77 Kelvin (K). The model has been used to understand the current interruption capability or arcing time of HTS breaker against switching voltage or current for different cases. The theoretical basis and the structure of the model that are deveped in this analysis are available in [6]. The model is validated on a sample of measured results. Three characteristics from the arc model are found and they are as follows: (i) The arc acts as a non-linear resistance. (ii) The power input for the arc channel recorded is nearly zero value. (iii) The arc voltage recorded is constant for the high current period [6].

\section{B. Controlled switching of HTS arc model}

The controlled switching of AC circuit breakers is a popular method to overcome the inrush current. However, there are some uncertainties in the measurement of circuit breaker's operating time [12]. CIGRE is noted to have good research works regarding the need of HVAC circuit breakers with controlled switching controller [13-15]. The method helps to reduce dielectric and thermal stresses during switching of shunt reactors, capacitors or transformers. Controlled switching has significant financial advantage along with several technical benefits. The performance of HTS breaker arc model has been used in this research with the controlled switching mode. This is vital to get steady operating time of HTS circuit breaker or breaker arc model throughout the operations. Usually, most of the modern era breakers present outstanding operating time regularity within $\pm 0.5 \mathrm{~ms}$.

\section{PARAMETER SWEEP OF HTS ARC MODEL}

It is frequently required to execute multiple iterations of an arc circuit to recover its faulty features. A parameter sweep of a complex circuit like arc model can be executed for a different variation of analysis frequencies. It can be used in order to estimate unknown model parameters, tune control parameters or to examine the effectiveness of an algorithm. The problem of minimizing the arcing time of HTS arc model with a parameter sweep is discussed in this section. A standalone executable MATLAB ${ }^{\circledR}$ based program is performed in this regard. Steps to perform parameter sweep in MATLAB are as follows:

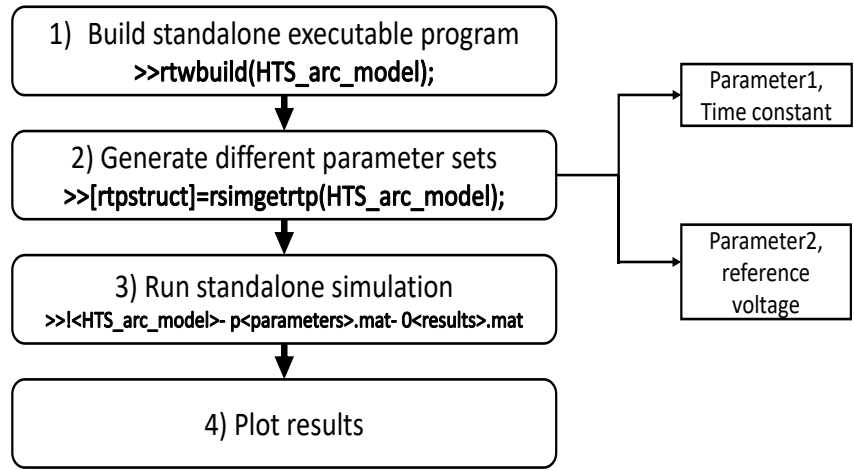

Figure 2: Parameter sweep steps of HTS arc model 
In Figure 2, the steps of parameter sweep are shown. First, a standalone executable program is built for the HTS arc model. Then, parameter set for the arc model is generated for the parameters like time constant and reference voltage. The iteration process shifts the dimensions of the parameters of arc model. The optimized value of parameterization provides the best results for minimum arc timing within a user defined range. Finally the results are shown by running the standalone simulation. The results are then analysed and compared from each run. The results between simulations change for different parameters used for parameter sweep. In order to repeatedly change the value of black box parameter, separate variable needs to be created in MATLAB workspace.

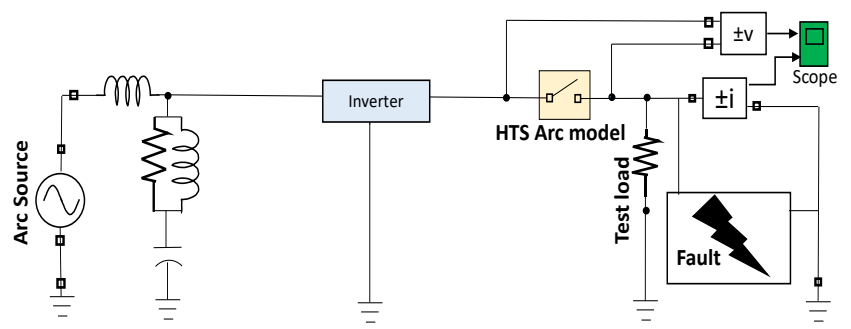

(a)

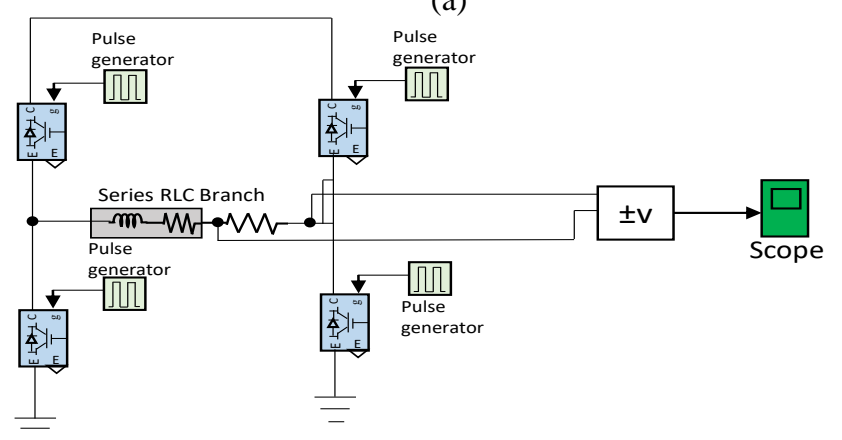

(b)

Figure 3: (a) Testbed circuit in Simulink. (b) Inverter for the arc model

In Figure 3(a), a test bed circuit in Simulink and an inverter is shown. Figure 3(b) shows the inverter used for the HTS arc model. In Table 1, the parameters for the test bed specification are enlisted.

TABLE 1. Test bed system specification

\begin{tabular}{|l|l|}
\hline Parameters & Value \\
\hline AC Supply voltage & $11 \mathrm{kV}$ \\
\hline Arc current & $1200 \mathrm{Amp}$ \\
\hline
\end{tabular}

\section{A. Parameter sweep for arc parameters}

If the arcing voltage is higher than reference voltage, the value of arc current cut down to zero. The simulation result of Browne's arc model can validate this scenario with parameter sweep. Arcing voltage time is decided for this by a time constant which ensures the dependency of fault time on selected parameters. In this analysis, the two parameters of Browne's arc model: reference voltage and time constant are selected for parameter sweeping calculation. For the iterative simulation the chosen sweep values for each parameter are shown in Table 2 and the result is shown against arc voltage and current.

TABLE 2. Iterative simulation of chosen Sweep values

\begin{tabular}{|l|c|l|}
\hline Parameters & $\begin{array}{c}\text { Default } \\
\text { value }\end{array}$ & Sweep value \\
\hline$\tau[\mu \mathrm{s}]$ & 100 & $50,80,110,140,170$ \\
\hline $\mathrm{U}_{\mathrm{c}}[\mathrm{kV}]$ & 11 & $9,11,13,15,17$ \\
\hline
\end{tabular}

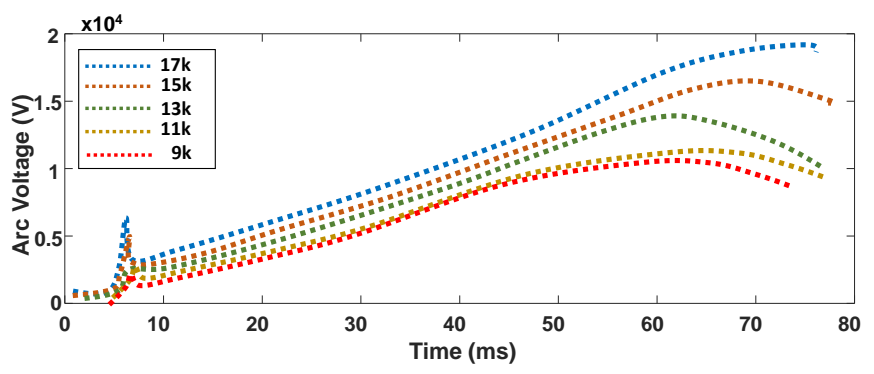

(a)

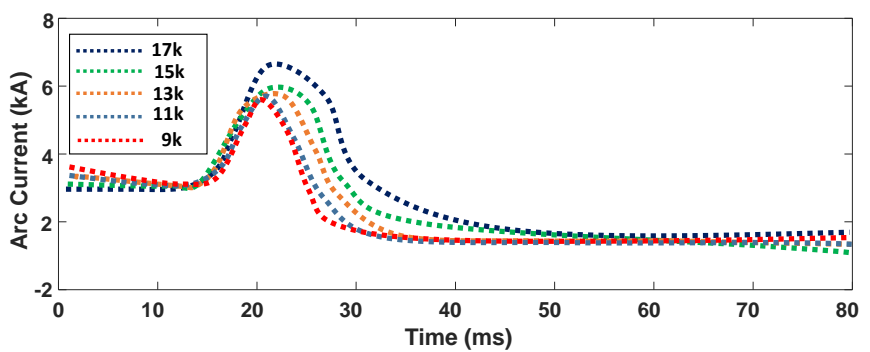

(b)

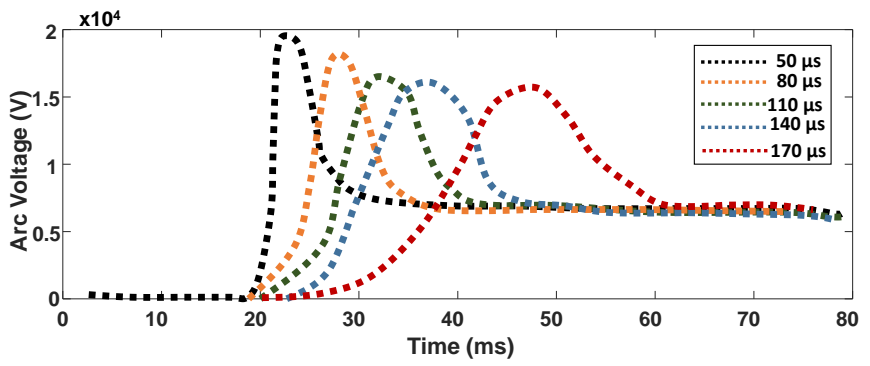

(c)

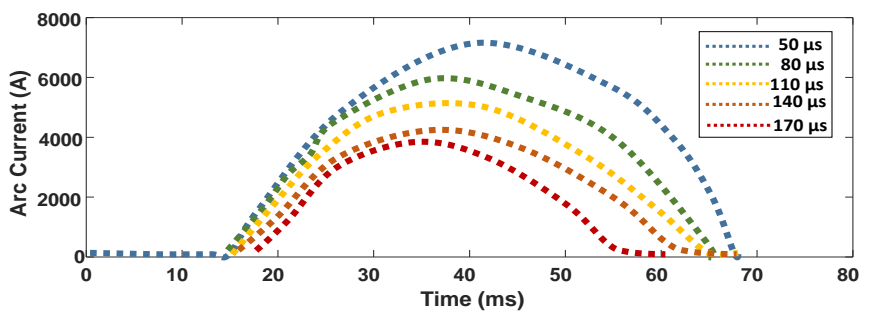

(d)

Figure 4: Browne's model transient response for parameter sweep

Figures 4(a) and 4(b) show the arc model's transient response by reference voltage. In Figure 4 (a), it is shown that when rated voltage $U_{c}$ is grown, the arc peak voltage also grows. It means if the reference voltage develops, the slope of voltage increases as well. Figures 4(c) and 4(d) show arc model's transient response by time constant. Figures 4 (c) and 4(d) 
demonstrate that time constant grows during initial stage and saturated with time. One can conclude that when the time constant increases, the voltage generation slow down. Consequently, the current interruption time slows down as well. The ignition becomes steeper to ensure arc current can reach zero instantly. It is noticeable, the time constant influences the arc voltage and current. If the time constant increases, the voltage profile stretches wider with transition and the current profile becomes broader. The arc voltage influences the voltage profile as well. The voltage profile follows an upward trend with the rise of the value.

\section{B. Parameter sweep for arc resistance}

The arc originates from the contacts or from the transition of high voltage discharge. For steady state situation, the rate of arc current changes very little. As an increased arc current flow for HTS contacts increase the ionization in Liquid Nitrogen (LN) arc chamber. Arc possesses negative resistance characteristics. Applied voltage and arc resistance may vary to calculate the property of arcing length.

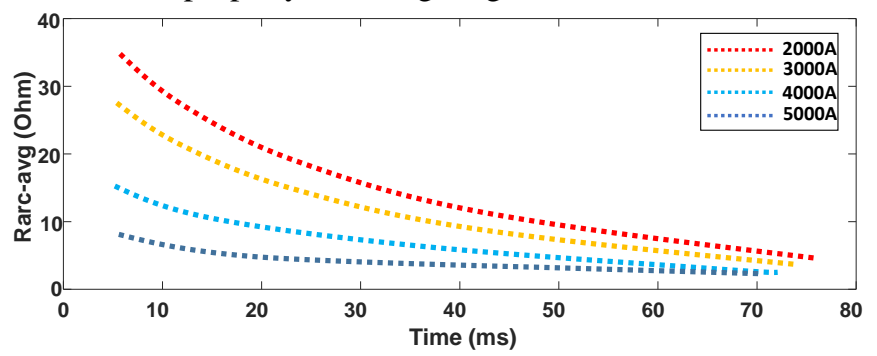

Figure 5: Average arc resistance

Fig. 5 demonstrates the average arc resistance for various current ranges. The arc resistance is calculated by dividing arc voltage with arc current at each data point. As a result arc's dynamic characteristic, the arc resistance is found as constant as a standard resistor. The graph is obtained by averaging the arc resistance for several gap lengths at described load current level. It is found that the arcing current has impact on arc resistance. When the current is low, the load current shows a significant impact on the arc resistance.

\section{COMPARISON OF ARC RESUlts}

The proposed HTSCB phenomenon is tested to investigate its arcing time for different current interruption cases. The interruption can be made for very restricted number of times as large power experiments are costly. The verification of the study is carried out with respect to time with comparison result of experimental setup and HTS arc model. The test circuit is shown in Figure 6. It has three major sections: (1) arc circuit (2) liquid nitrogen arc chamber and (3) measurement unit. In section1 of Figure 6, an arc circuit with 700-1200 ampere arc source is used to provide high peak current within millisecond range. The circuit is attached for producing the arc at the source side of the HTSCB. Section-2 of Figure 6 is the liquid nitrogen (LN) arc chamber where the arcing happens. The HTS contacts are submerged into LN and the movable HTS contact is travelled 10$25 \mathrm{~mm}$ with the help of a tension spring. The moveable contact is an HTS pull-rod (CSL-26/120.3 type) and the fixed contact is made of Yttrium barium copper oxide (YBCO) which is attached to centre of the base plate of cryogenic arc chamber. In section-3 of Figure 6, the numerical values of the measurement results are recorded in measurement unit. 1252C RCD meter is used to measure the arcing time. 34972A-Data logger switch unit and USB 2404 series measurement computing is used to measure numerous temperature and pressure reading.

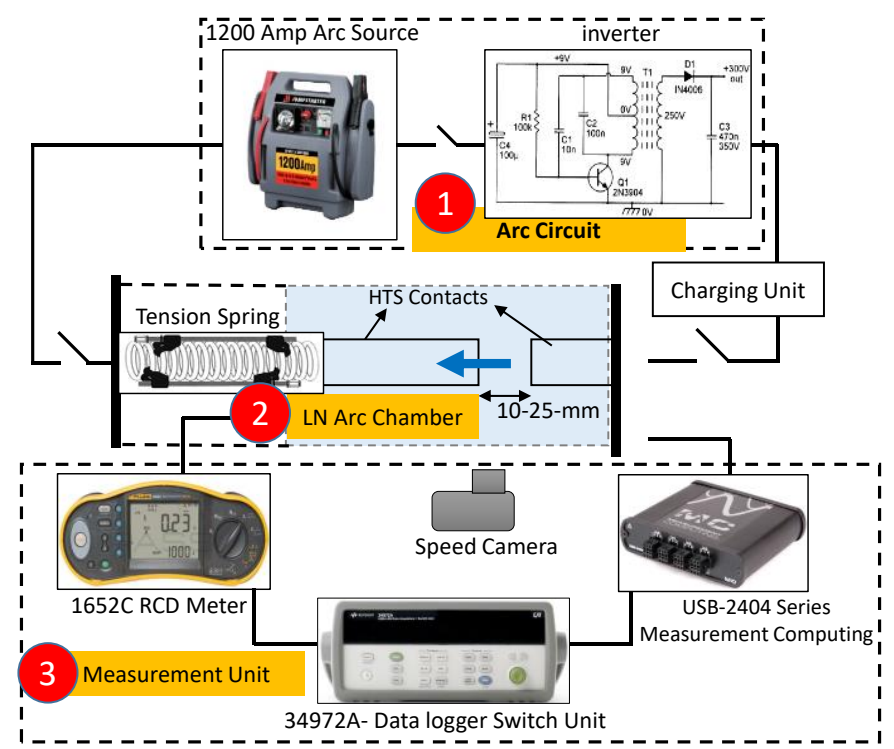

Figure 6: Test object configuration and overview of experimental set-up for arc phenomena captured with thermographic speed camera in cryogenic arc chamber

In Figure 7(a) four test results from the experimental set us are plotted and then compared with HTS arc model's simulation arc result in Figure 7 (b). It is found that the arc current test result waveform have similarity with arc model's arc current wave form. In experimental result the arc timing varied from 50 to 80 milliseconds, while in simulation result it was much consistent near 50 to 60 milliseconds.

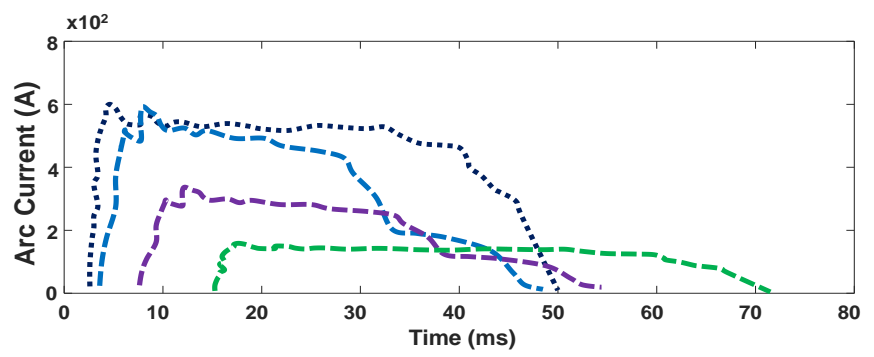

(a) 


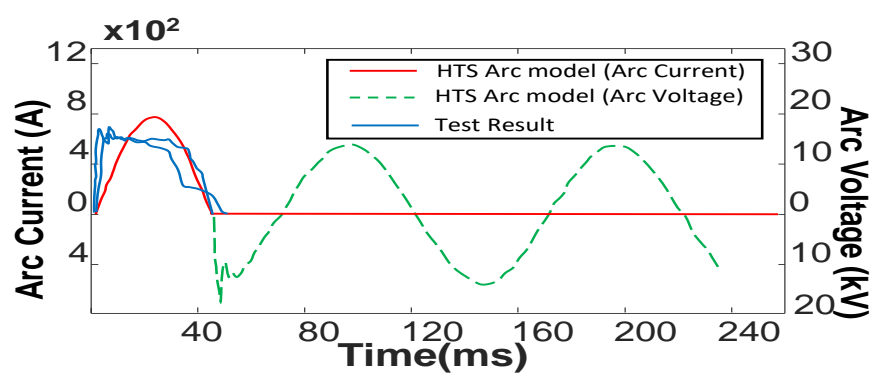

(b)

Figure 7: (a) Test results from experimental set up (b) Comparison of test and simulation results

However, a big deviation for fault current is observed between the results. For the same fault current of 700A the current was recoded up to $800 \mathrm{~A}$ in HTS arc model simulation result. The precision for arcing timing is expected to develop during real time implementation of arc model.

TABLE 3. Arc characteristic in experimental model

\begin{tabular}{|c|c|c|}
\hline Arc peak (A) & $\begin{array}{c}\text { Post arc } \\
\text { current peak } \\
(\mathrm{A})\end{array}$ & $\begin{array}{c}\text { Rate of post- } \\
\text { arc current } \\
(\mathrm{A} / \mu \mathrm{s})\end{array}$ \\
\hline 1200 & 5 & 2.7 \\
\hline 1200 & 15 & 2.1 \\
\hline 1200 & 12 & 3.3 \\
\hline
\end{tabular}

In Table 3, the arc characteristic reading for the experimental model is listed. The experiments have shown stability of minimum arcing times showing important potential for HTSCB design optimization.

\section{VI, CONCLUSION}

In this paper, HTS black box arc model is reviewed for a test bed scheme in order to develop better understanding about interruption behavior of HTSCB. Two fundamental arc models are utilized to simulate the transient behaviors of circuit breaker under opening status. Experimental studies were conducted to verify the developed model. The results from both simulation and experimental studies were in agreement. Thus, the proposed model is fairly accurate. The future research will be focused on identification of different failure scenarios and their reasons using intelligent methods. Automatic classification algorithms can be effective in order to achieve this goal.

\section{ACKNOWLEDGMENT}

The authors are grateful for the support to School of Engineering, Computer and Mathematical Sciences, Auckland University of Technology, New Zealand.

\section{REFERENCES}

[1] Stuart C. Wimbush, Large Scale Applications of HTS in New Zealand, Physics Procedia, Pages 221-224, ISSN 1875-3892, Volume 65, 2015.

[2] Hu, Daoyu, M. Hu, Y. G. Xu, X. F. Sheng, J. N. Li, Zhuyong Li, Zhiyong Hong, and J. Jin. "Design and Electromagnetic Analysis of a 330 kVA SinglePhase HTS Transformer." IEEE Transactions on Applied Superconductivity 27, no. 4 (2017): $1-5$
[3] Hu, Daoyu, Zhuyong Li, Zhiyong Hong, and Zhijian Jin. "Development of a single-phase 330kVA HTS transformer using GdBCO tapes." Physica $C$ : Superconductivity and its Applications 539 (2017): 8-12.

[4] Sjostrom, Marten, and Diego Politano. "Technical and economical impacts on a power system by introducing an HTS FCL." IEEE transactions on applied superconductivity 11, no. 1 (2001): 2042-2045.

[5] C. W. Bumby, A. E. Pantoja, H. J. Sung, Z. Jiang, R. Kulkarni and R. A. Badcock, "Through-Wall Excitation of a Magnet Coil by an External-Rotor HTS Flux Pump," in IEEE Transactions on Applied Superconductivity, vol. 26, no. 4 , pp. 1-5, June 2016.

[6] Ullah, A., T. T. Lie, K. Gunawardane, and N. K. C. Nair. "Development of browne's arc model for HTS applications." In Power System Technology (POWERCON), 2016 IEEE International Conference on, pp. 1-4. IEEE, 2016.

[7] Ullah, A., T. T. Lie, K. Gunawardane, and N. K. C. Nair. "The improvement of Rate of Rise of Recovery Voltage (RRRV) for an HTS breaker " In 12th IEEE PES Powertech Conference, IEEE, 2017

[8] D. Djurek, Z. Medunić, A. Tonejc, and M. Paljević, "PbCO3·2PbO+Ag2O and $\mathrm{PbCO} 3 \cdot \mathrm{PbO}+\mathrm{Ag} 2 \mathrm{O}$ (PACO) systems: route for novel superconductors," Physica C: Superconductivity, vol. 351, p. 4, March 2001.

[9] R. Wesche, High-Temperature Superconductors: Materials, Properties, and Applications. Boston/Dordrecht/London: Kluwer Academic Publishers, 1998.

[10] B. W. McConnell, S. P. Mehta, and M. S. Walker, "HTS transformers," Power Engineering Review, IEEE, vol. 20, pp. 7-11, 2000.

[11] Ebner, A. "Determination of acceptable closing time scatter and residual flux measurement uncertainty for controlled switching of transformers." In Proceedings of the 16th international symposium on high voltage engineering, pp. 1-6. 2009.

[12] Controlled switching of HVAC circuit breakers: guide for application lines, reactors, capacitors, transformers: Part-I. ELECTRA 183, Cigré Working Group A3.07; April 1999. p. 43-73.

[13] Controlled switching of HVAC circuit breakers: planning, specification and testing of controlled switching systems. ELECTRA 197. Cigré Working Group A3.07; August 2001. p. 22-33.

[14] Controlled switching of HVAC circuit breakers: guide for application lines, reactors, capacitors, transformers: Part-II. ELECTRA 185. Cigré Working Group A3.07; August 1999. p. 37-57.

[15] Kulkarni, Shrikrishna V., and S. A. Khaparde. Transformer engineering:design and practice. Vol. 25. CRC Press, 2004.

[16] M. Young, The Technical Writer's Handbook. Mill Valley, CA: University Science, 1989

[17] P. Lin, J. Gu, M. Yang, "Intelligent maintenance model for condition assessment of circuit breakers using fuzzy set theory and evidential reasoning," IET Gener. Transm. Distrib., Vol. 8, No. 7, pp. 1244-1253, Jul. 2014.

[18] S. S. Biswas, A. K. Srivastava, D. Whitehead, "A Real-Time Data-Driven Algorithm for Health Diagnosis and Prognosis of a Circuit Breaker Trip Assembly," IEEE Transactions on Industrial Electronics, Vol. 62, No. 6, pp. 3822-3831, Jun. 2015.

[19] J. Tang, S. Lu, J. Xie, Z. Chengb, "Contact Force Monitoring and Its Application in Vacuum Circuit Breakers," IEEE Transactions on Power Delivery, Vol. PP, No. 99, pp. 1-1, Apr. 2015.

[20] A. A. Razi-Kazemi, M. Vakilian, K. Niayesh, M. Lehtonen, "Data Mining of Online Diagnosed Waveforms for Probabilistic Condition Assessment of $\mathrm{SF}_{6}$ Circuit Breakers," IEEE Transactions on Power Delivery, Vol. 30, No. 3, pp. 1354-1362, Jun. 2015.

[21] A. A. Razi-Kazemi, "Circuit breaker condition assessment through a fuzzy-probabilistic analysis of actuating coil's current," IET Gener. Transm. Distrib., Vol. 10, No. 1, pp. 48-56, Jan. 2016.

[22] Feizifar, Behnam, and Omer Usta. "Condition monitoring of circuit breakers using arc models and failure detection algorithm." In Smart Grid and Cities Congress and Fair (ICSG), 2017 5th International Istanbul, pp. 32-36. IEEE, 2017

[23] A. Ebner, "Determination of acceptable closing time scatter and residual flux measurement uncertainty for controlled switching of transformers." In Proceedings of the 16th international symposium on high voltage engineering, pp. 1-6. 2009.

[24] Controlled switching of HVAC circuit breakers: guide for application lines, reactors, capacitors, transformers: Part-I. ELECTRA 183, Cigré Working Group A3.07; April 1999. p. 43-73. 